\title{
Concentration at Detection Limit of Dopant for Semiconductor Samples Using Dual SDD Analysis System.
}

\author{
K. Fukunaga ${ }^{1}$, N. Endo ${ }^{1}$, M. Suzuki ${ }^{2}$ and Y. Kondo ${ }^{1}$ \\ ${ }^{1}$ JEOL Ltd., 3-1-2 Musashino, Akishima, Tokyo 196-8551, Japan \\ 2 Thermo Fisher Scientific Japan, 3-9 Moriya-cho, Kanagawa-ku, Yokohama, 221-0022, Japan
}

An energy dispersive spectrometer (EDS) analysis system using a Si drift detector (SDD) can enhance sensitivity of compositional analysis by using a large solid angle detector $[1,2]$. The use of multiple EDS detector system dramatically enlarge the effective area of the detector, resulting in the short measurement period. Up to now, transmission electron microscopy (TEM)-EDS has not been greatly used in the analysis of elements for semiconductor doping. This is because the detection limit by $\mathrm{Si}(\mathrm{Li})$ - EDS is of the order of 1500-2000 ppm, which is insufficient for semiconductor industry. There has consequently been a strong desire to see "limit of detection (LOD)", that is, how small an amount of elements we can be detected, using a multiple SDD analysis system. In this study, we explore the concentration at LOD for As dopant in Si device using FETEM with a dual SDD analysis system.

We used an As-doped Si wafer as a sample. The depth profile of As concentration in the Si wafer has been verified by Secondary Ion Mass Spectrometry (SIMS). We made a cross sectional lamella sample for TEM observation using an ion milling (Ion Slicer EM-09100IS, JEOL). The thickness of the TEM sample is estimated to be $140 \mathrm{~nm}$ judging from the count rate of X-ray and probe current. We measured several data sets of EDS spectrum imaging using a 200kV FETEM (JEM-2800 JEOL) equipped with two SDD detectors (sensor area $=100 \mathrm{~mm}^{2}$ ). The concentration profiles were extracted and converted from the data set with a quantitative analysis system (NSS 3, Thermo Fisher Scientific).

Figure 1 shows cross sectional STEM-HAADF image of the sample. The yellow, brown and blue rectangles show the regions of analyzed, As rich and pure Si. The locations for As rich and pure Si were determined from As depth profile of SIMS. Figure 2 shows EDS spectra from the brown rectangle region. We can see the peaks for As-K and As-L. Figure 3 shows concentration profiles of As in Si using As-K and As-L lines. Theoretical estimation of LOD is determined with competition between the net counts for dopant element and the fluctuation of background tail of Si peak at the dopant peak energy. If we take the net counts of dopant element is three times larger than the fluctuation of the background, which confirms the presence of the dopant with $99.7 \%$ certainty, we define an approximate dopant concentration $\left(C_{\text {dopant }}\right)$ at LOD as

$$
C_{\text {dopant }}=\frac{3 k}{\sqrt{I_{n e t} \times P / B}} \ldots \text { (1) }
$$

,where the $I_{\text {net }}$ is net count of $\mathrm{Si}-\mathrm{K}$, the $P / B$ is ratio of net count of $\mathrm{Si}(P)$ and background count at energy of As lines $(B)$, and $k$ is $\mathrm{K}$ factor for lines of dopant, which is unity in this calculation. The theoretical $C_{d o p a n t}$ are calculated to be $30 \mathrm{ppm}$ for As-K and $80 \mathrm{ppm}$ for As-L from equation (1). These are value of blue lines (1nA, $80 \mathrm{~min}$ ) shown in Fig. 3(a) and 3(b). The values for the calculation are listed as $\mathrm{I}_{\text {net }}=$ $6,800,000, \mathrm{P} / \mathrm{B}$ for As-K $=1,480, \mathrm{P} / \mathrm{B}$ for As-L $=220$. The $C_{\text {dopant }}$ for As-K is smaller than that for As-L, although net count of As- $\mathrm{L}=9,800$ is larger than that of As-K $=5,100$. This is due to that the P/B of As-K is better than that of As-L. Therefore, in order to increase the sensitivity, it is important not only to increase the number of counts, but it is effective to choose a line of X-ray having a high P/B. 
References:

[1] S. Kawai, I. Ohnishi, T. Ishikawa, K. Yagi, T. Iwama, K. Miyatake, Y. Iwasawa, M. Matsushita, T. Kaneyama and Y. Kondo, Microscopy \& Microanalysis 20 (S3) (2014), p. 1150-1151.

[2] I. Ohnishi, S. Kawai, T. Ishikawa, K. Yagi, T. Iwama, K. Miyatake, Y. Iwasawa, M. Matsushita, T. kaneyama and Y. Kondo, Proceedings of IMC18 (Prague 8-12 Sept.) (2014).

[3] This equation is equivalent listed in "Introduction to Analytical Electron Microscopy" (ed. J J Hren, J I Goldstein and D C Joy, Prenum Press) Chap.3 (1979) p.113 with 3 times standard deviation.

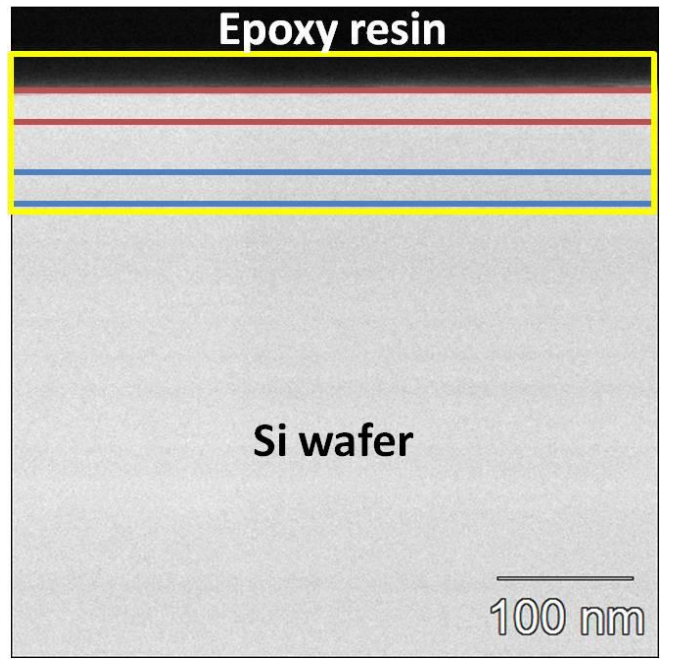

Figure 1. STEM-HAADF image of cross sectional sample made from As-doped Si wafer. (a)

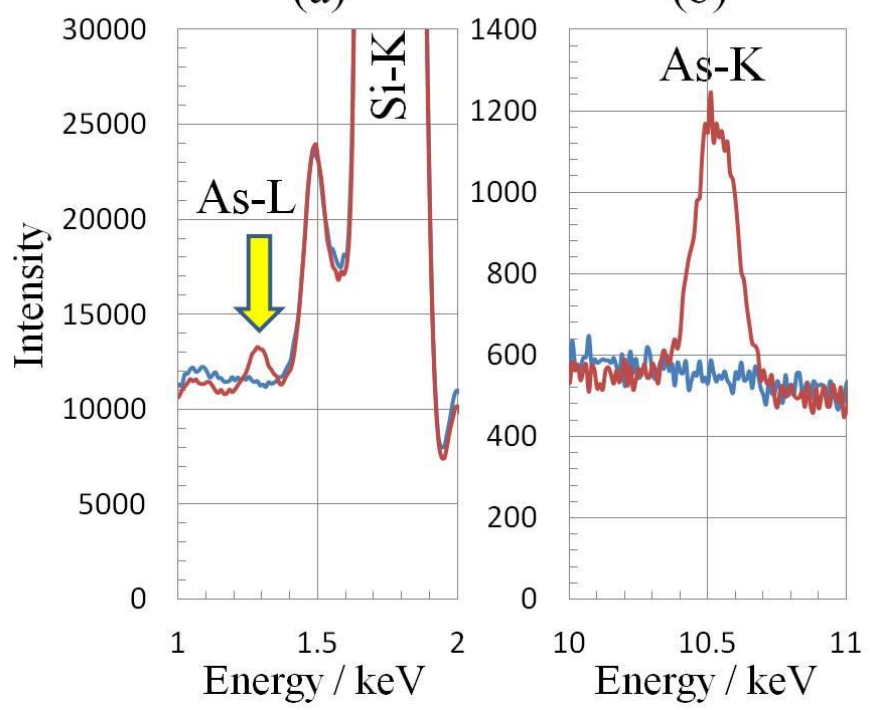

Figure 2. EDS spectra from the brown rectangular region in Fig. 1. (a) As-L, (b) As-K.
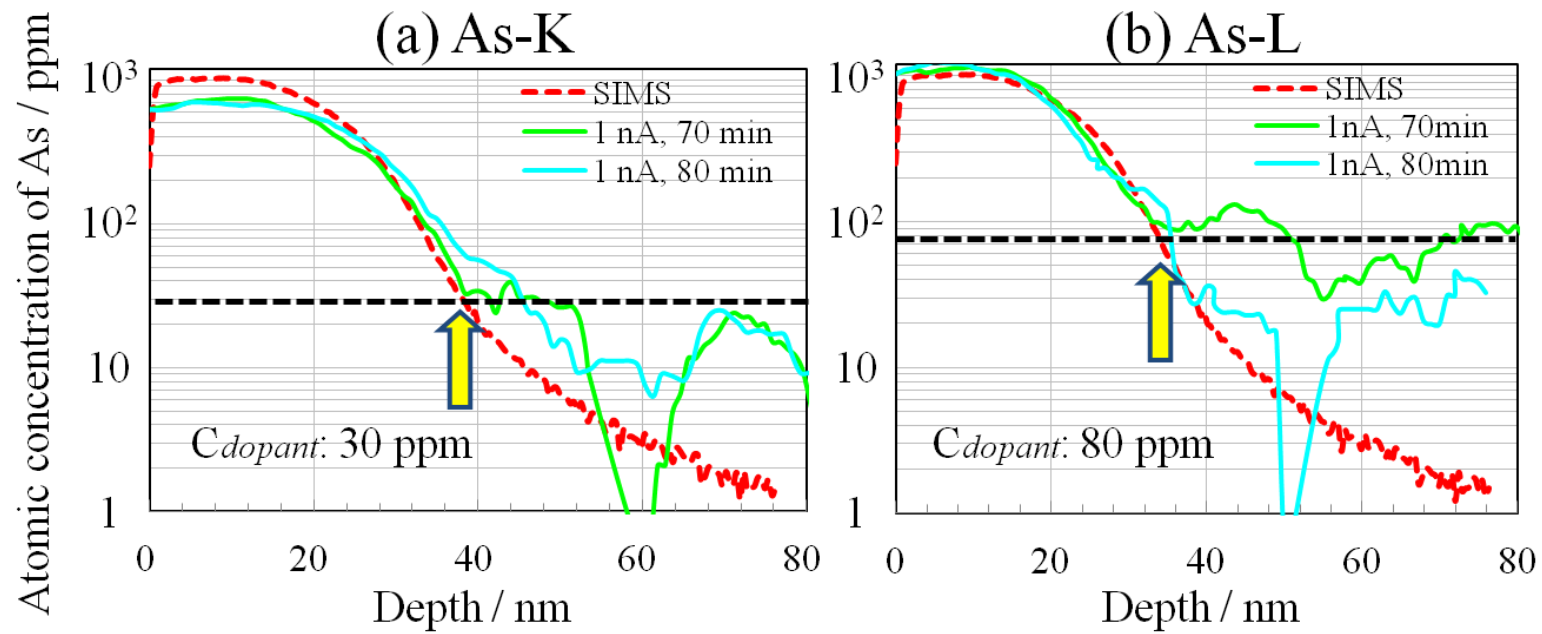

Figure 3. Profiles of As concentration in Si using (a) As-K and (b) As-L lines. The yellow arrows are theoretical estimation of $C_{\text {dopant }}$ at LOD (blue line). Red lines indicate the concentration profile measured with SIMS. 